\title{
New, Binomial Model of Atom, Trajectories and Schemes of Energy of Splitting Levels of Atom
}

\author{
Alexander V. Yurkin \\ City of Puschino, Russia
}

\begin{abstract}
Offered work, it is devoted to a geometrization of physics and research of ray system. We consider system of circular, wavy and zigzag trajectories of the movement of electrons in atom shells. We show communication between the nuclear model of atom and wavy trajectories located along a horizontal tape. The offered model of the movement of electrons is based on geometrical interpretation of the principle of Pauli and Periodic Table of chemical elements. Visual interpretation of distribution of electrons in energy levels and shells of atom is shown. In our constructions we are also based on the known models of trajectories of electrons in atom. We also demonstrated calculations illustrate the splitting of the energy levels of the atom by introducing an additional parameter.. It has turned out that the known schemes of energy levels of atom received from experimental spectral studies can be also received in our binomial model from geometrical creation of the main and azimuthal quantum numbers by easy ways, for example, by means of compasses, a ruler, and elementary calculations. Our approximate geometrical model can visually add the known geometrical and physical models explaining a structure of atoms.
\end{abstract}

Keywords: atom, binomial, trajectories, energy splitting levels, geometrization of physics

\section{INTRODUCTION}

1.1. The radiation of atoms consists of spectral lines. Experimental studying of spectrum of atoms serves as knowledge of a structure of atoms [1 - 4]. On the basis of experimental spectral studies the energy level schemes of atoms explaining their structure are under construction. Visual geometric models are widely used for the description of different phenomena in the nature [5]. The periodic table of chemical elements (Tables 36.1 and 37.1 from [3] or Figs. 24 and 25 from [10]) can be seen as the visual model describing properties of atoms too. Such periodic table isn't single. In [6] it is shown that periodic regularities of chemical elements can be visually presented by tables in the form of circulars, pyramids, spirals etc. The nuclear model of atom in which atom consists of a nucleus and electron shells was offered by Rutherford. Geometrical planetary models of a structure of atom were offered by Bohr in the form of circle orbits of movement of electrons around a nucleus and Sommerfeld [7] in the form of elliptic orbits. In monographs [2 - 4] different models and configurations of electronic orbits and electronic clouds are provided (Figs. 228, 230, 250, 255, 257 from [2], and Fig. 23 from [4]). However, these models don't explain creation of energy level schemes of atom clearly (Figs. 28.1, 29.1, and 31.1 from [3]) which were received from the experimental studies of spectral lines of atom.1.2. In work [8] binomial distribution of new type was offered. In work [9] visual geometrical interpretation of processes in long tubes has been described. Visual geometrical models laminar and turbulence of liquid in tubes, findings of an electron in infinitely deep potential well and distribution of light in the laser are presented in works [9] and [10].Work [10], has been devoted to a research of half-integer ray system, twodimensional and three-dimensional geometric models of the movement of particles with half-integer spin. In work [10] the new geometrical model of a structure of electron shells of atom has been offered and geometrical interpretation of the main, azimuthal, magnetic and spin numbers in the form of angles and distances is given, shells and subshells of atoms are interpreted as systems of the wavy trajectories consisting of direct inclined pieces. In work [10] on the basis of consideration of the Principle of Pauli and our geometrical constructions we have drawn a conclusion that atom may contain no more than 8 electron shells, i.e. these are shells of $\mathrm{K}, \mathrm{L}, \mathrm{M}$, $\mathrm{N}, \mathrm{O}, \mathrm{P}, \mathrm{Q}$ and R, otherwise shells will be imposed at each other. In work [11] the geometrical model of the second shell of atom has been separately considered, communication of this model with the cubic equation and a formula of Cardano is shown, the special type of a triangle is described. 1.3. In the offered work we conduct further researches of the geometrical model of atom and its electron shells offered in work [10]. We show communication between the nuclear model of atom and wavy trajectories located along a horizontal tape. We adhere to geometrical and recurrent approach for the approximate description of power levels of atom proceeding from nuclear model of atom, Pauli's principle and the Periodic Table. We show in this work that the known schemes of energy levels of atom received from experimental spectral studies can be also received in our binomial model from geometrical creation of the main and azimuthal quantum numbers by easy ways, for 
example, by means of compasses, a ruler, and elementary calculations. In creation of our new approximate model we are also based on the known models of trajectories of electrons in atom.From history of mathematics it is known that the probability theory which is widely applied in various areas for example in quantum mechanics [1 - 4] has arisen including from consideration of binomial distribution [12]. Binomial distribution can be received from an arithmetic triangle of Pascal [12] which geometrical creation was known in ancient India and China.Evident geometrical schemes allow represent at first sight difficult systems obvious without additional proofs and assumptions. I. Newton's statement is widely known that it is optional to prove Euclid's theorems as they so are obvious. In this work we show that our evident geometrical binomial model doesn't contradict Paulie's principle, the Periodic Table and probability theory. Ratios between separate elements of our model can be interpreted as a trajectory of various forms, as quantum numbers and as the power levels received from experimental spectral studies We also demonstrated calculations illustrate the splitting of the energy levels of the atom by introducing an additional parameter. We hope that our new visual binomial geometrical model of atom will be able to add the geometrical and physical models of atom which are listed above in item 1.1 Geometrization of physics will help to enrich even more quantum mechanics and to make it more available to understanding.

Numerical calculations, in this work, as well as in works [9 - 11], we carried out in the MS Excel program.

\section{WAVY AND CIRCULAR TRAJECTORIES OF THE MOVEMENT OF ELECTRONS IN ATOM}

\subsection{Paraxial binomial system of trajectories}

In works $[9,10]$ we have described paraxial binomial (sharing in two) flat system of trajectories. Здесь мы уточним наш подход. This system consists of groups of rays in which rays are inclined under $p$ angles small to an axis and multiple to an angle $\gamma$ :

where

$$
p=(m+1 / 2) \gamma
$$

$$
m=0, \pm 1, \pm 2 \ldots
$$

Let us accept, in addition, that:

where

$$
p_{0}=\left( \pm \frac{1}{2}\right) \gamma=s \gamma,
$$

for

$$
s= \pm \frac{1}{2}
$$

$$
m=0 \text {. }
$$

Points of branching of rays will be spaced from a symmetry axis on small distances of $q$ multiple to $k / 2$ length:

where

$$
q=j k / 2 \text {, }
$$

$$
j=0, \pm 1, \pm 2 \ldots .
$$

We called this system of rays: "[p=(m+1/2) $\gamma, q=j k / 2]$ - system" or half-integer ray system. This binomial system of rays consists of groups of rays $\mathbb{N}$ and their links $\mathbb{K}[10]$.

In this work, also as well as in the previous works $[9,10]$ we assume that rays, have energy of $\boldsymbol{E}$ and extend along the branching $\mathbb{K}$ links therefore the number of rays of $\mathbb{N}$ (and their energy $\boldsymbol{E}$ ) can be summarized. Generally $\mathbb{N} \geq \mathbb{K}$. We designate number of passes of rays along links as $0,1,2 \ldots, \mathrm{n}-1, \mathrm{n}, \mathrm{n}+1 \ldots$

This system of rays is placed in a rectangular coordinate grid. The size of a cell of a grid has height of $k / 2$ and length of $L$. In work [10] we have shown what various configurations of our flat system of the trajectories consisting of rays corresponds to distribution of electrons in various electron shells of atom according to Paulie's principle and the Periodic Table.

\subsection{Images of shells $\mathrm{K}, \mathrm{L}, \mathrm{M}$ and $\mathrm{N}$ of atom}

Process of consecutive visual geometrical creation of shells and subshells of atom in the form of the wavy trajectories extended along horizontal tapes has been presented in work [10]. In this work we show how such trajectories are connected with the image of circular orbits of electrons in atom.

In Fig. 1 the first four electron shells $\mathrm{K}-\mathrm{N}$ of atom for our model are represented. In Fig. 1a circular orbits are represented, in Fig.1b these orbits are extended in the form of tapes along a horizontal. Actually, the image in Fig.1b is conformal display of the image submitted in Fig.1a [13]. In Fig.1c - $\mathrm{f}$ are shown the broken wavy trajectories extended along horizontal tapes for completely filled electron shells $\mathrm{K}-\mathrm{N}$ respectively. In Fig.1g - j are shown the circular broken orbits corresponding to wavy trajectories in Fig. 1c $-\mathrm{f}$. The images submitted in Fig.1 can be constructed by easy ways, for example, by means of compasses, a ruler, and elementary calculations. 

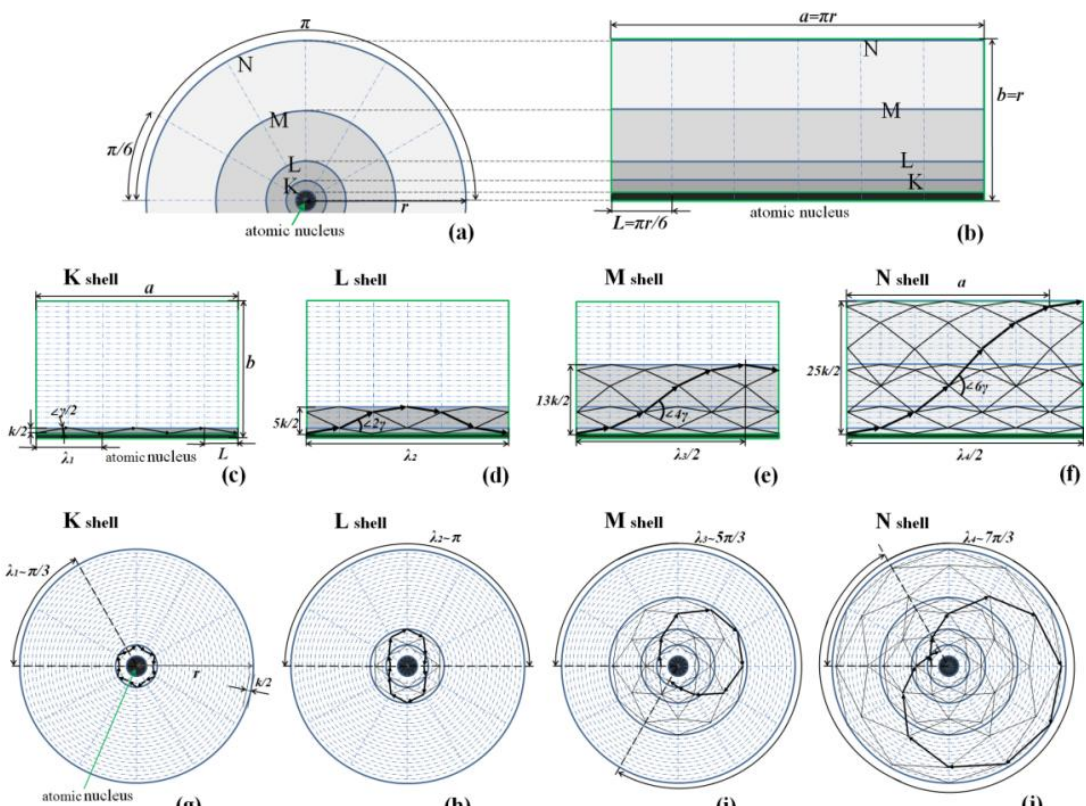

(g)

(h)

(i)

(j)

Fig1. First four shells of atom. (a) Nuclear model of atom, the image of nuclear and circular orbits of electrons (coincides with Fig.228 from [2]). (b) Display of nuclear model of atom to a horizontal tape. (c - $f$ ) Completely filled atomic shells of $K-L$ in the form of wavy trajectories extended along horizontal tapes. $(\boldsymbol{g}-\boldsymbol{j})$ Display wavy horizontal trajectories on the corresponding circular trajectories. (g) A circular wavy trajectory (corresponds to six "waves" $\lambda_{1}$ represented on (c) it is similar to the trajectory represented in Fig.27 from [4]. (h) Dumbbell-shaped a trajectory (corresponds to two "waves" $\lambda_{2}$ represented on $(\boldsymbol{d})$, it is similar to the images in Fig.230 and Fig.250 from [2]. ( $i$ and $j$ ) The penetrating and precessing trajectories (correspond to one "wave" $\lambda_{3}$ or $\lambda_{4}$ represented on (e or f)) are similar to trajectories represented in Fig. 255 or Fig.257 from [2], etc. Heavy shooters have allocated longest "waves".

\subsection{Images of shells $O, P, Q$ and $R$ of atom}

In Fig. 2 other four electron shells $\mathrm{O}-\mathrm{R}$ of atom, and also $\mathrm{N}$ shell (in the reduced scale in comparison with Fig.1) for our model are represented. In Fig.2a circular orbits are represented, in Fig.2b these orbits are extended in the form of tapes along a horizontal. In Fig. $1 \mathrm{c}-\mathrm{f}$ are shown the broken wavy trajectories extended in the form of horizontal tapes for completely filled electron shells O - R.

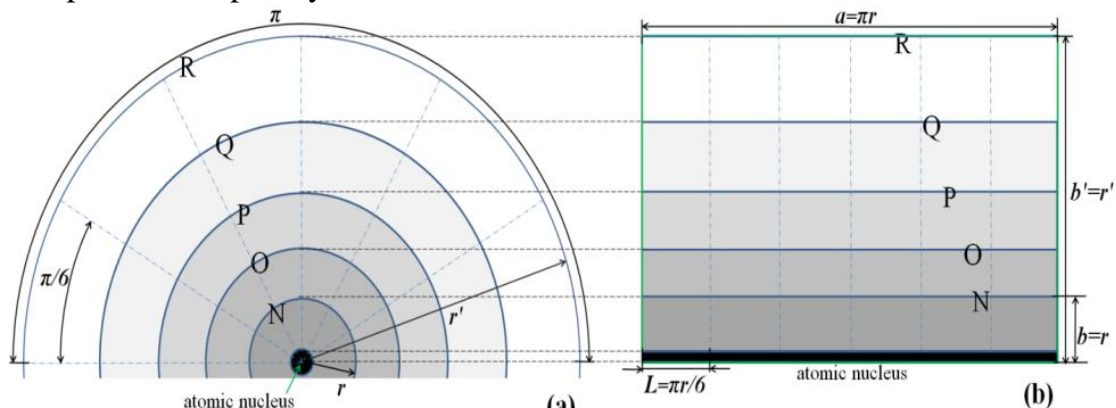

atomic nucleus

(a)

(b)

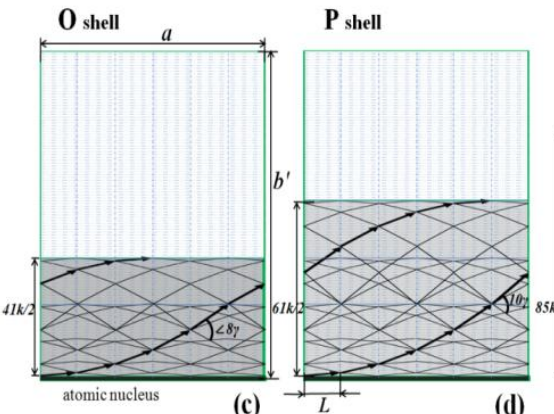

(d)
Q shell

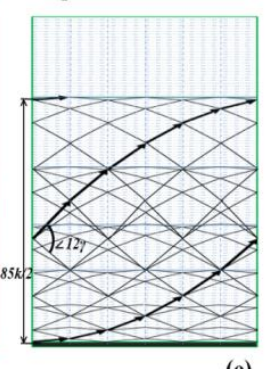

$\mathbf{R}$ shell

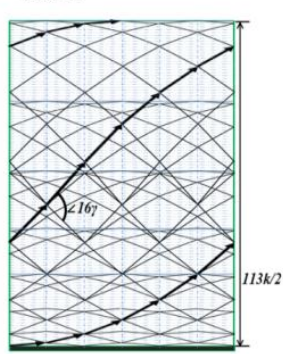

(f) 
Fig2. 4th - 8th atomic shells. (a) Nuclear model of atom, images of a nuclear and circular orbits of electrons. (b) Display of nuclear model of atom to a horizontal tape. (c - $f$ ) Completely filled atomic shells $O-R$ in the form of the wavy trajectories extended along horizontal tapes; heavy shooters have allocated longest "waves".

\section{GEOMETRICAL CREATION OF THE SCHEME OF ENERGY LEVELS OF QUANTUM SYSTEM}

3.1. In work [10] for calculation of shells of atom we used the three-dimensional arithmetic table an arithmetic parallelepiped. Such approach has allowed us to show wavy trajectories visually.

In this work we will use other approach for designation of rays in our binomial model to show geometrical creation of the scheme of the energy levels of atom received from spectral researches [1 - 4] (Figs. 28.1, 29.1, and 31.1 from [3], and/or Fig. 28 from [10]).

Let us enter the following designations:

$$
\boldsymbol{E}(\boldsymbol{n}, \boldsymbol{l})^{\prime}
$$

it is the energy extending along the ray characterized by numbers $\boldsymbol{n}$ and $\boldsymbol{l}$ at each odd pass of $\mathrm{n}$ and

$$
\boldsymbol{E}(\boldsymbol{n}, \boldsymbol{l})^{\prime \prime}
$$

it is the energy extending along the ray characterized by numbers $\boldsymbol{n}$ and $\boldsymbol{l}$ at each even pass of $\mathrm{n}-1$ or $\mathrm{n}+1$. Here $n-1, n$ and $n+1$ are numbers of passes of rays. Numbers $\boldsymbol{n}$ and $\boldsymbol{l}$ characterize each of rays of our binomial system.

Let us designate average energy within two neighbor passes:

Generally numbers $\boldsymbol{n}$ and $\boldsymbol{l}$ accept values

$$
E(n, l)=\left[E(n, l)^{\prime}+E(n, l)^{\prime}\right] / 2
$$

$$
n=1,2,3, \ldots
$$

and

$$
l=0,1,2, \ldots
$$

\subsection{First three shells of quantum system.}

In Fig.3 the first three atomic shells are represented (similar to Fig.1c - e) completely filled atomic shells.

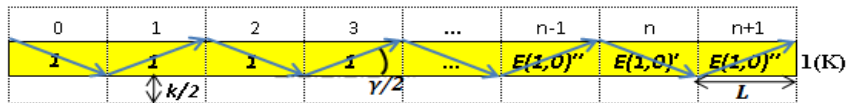

(a)

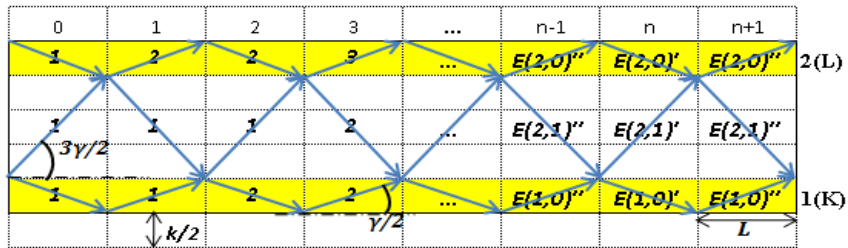

(b)

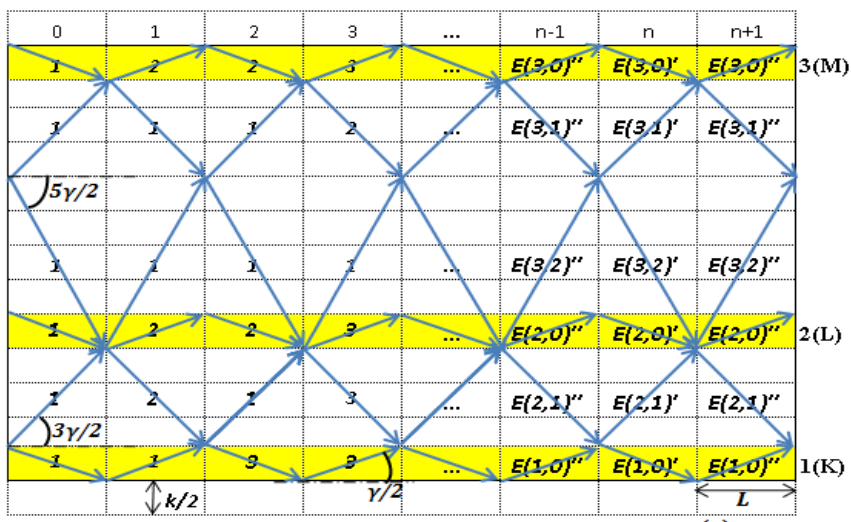

(c)

Fig3. Images of wavy trajectories of the first three completely filled atom shells. (a) The first $K$ shell, consists of the links inclined on angles $\gamma / 2$ to a horizontal; energy of $\boldsymbol{E}(\boldsymbol{n}, \boldsymbol{l})$ ' and $\boldsymbol{E}(\boldsymbol{n}, \boldsymbol{l})$ " which extends along these links are characterized by numbers of $\boldsymbol{n}=\mathbf{1}$ and $\boldsymbol{l}=\mathbf{0}$. (b) The second L cover, consists of the links inclined on angles $\gamma / 2$ and $3 \gamma / 2$ to a horizontal; energy of $\boldsymbol{E}(\boldsymbol{n}, \boldsymbol{l})$ ' and $\boldsymbol{E}(\boldsymbol{n}, \boldsymbol{l})$ " which extends along these links are characterized by numbers of $\boldsymbol{n}=\mathbf{1}, 2$ and $\boldsymbol{l}=\mathbf{0 , 1}$. (c) The third $M$ cover, consists of the links inclined on 
angles $\gamma / 2,3 \gamma / 2$ and $5 \gamma / 2$ to a horizontal; energy of $\boldsymbol{E}(\boldsymbol{n}, \boldsymbol{l})^{\prime}$ and $\boldsymbol{E}(\boldsymbol{n}, \boldsymbol{l})$ " which extends along these links are characterized by numbers of $\boldsymbol{n}=\mathbf{1}, \mathbf{2}, \mathbf{3}$ and $\boldsymbol{l}=\mathbf{0}, \mathbf{1}, \mathbf{2}$.

\subsubsection{First $K$ shell of atom}

The energy extending along rays of our system in the first $\mathrm{K}$ shell of atom (Fig.3a) at pass of $\mathrm{n}$ is calculated as:

At pass of $n+1$ :

$$
E(\mathbf{1}, \mathbf{0})^{\prime}=E(\mathbf{1}, \mathbf{0})^{\prime \prime} \text {. }
$$

$$
E(1,0)^{\prime \prime}=E(1,0)^{\prime}
$$

where $\boldsymbol{n}=\mathbf{1}$ and $\boldsymbol{l}=\mathbf{0}$. And average energy for two passes:

$$
E(1,0)=\left[E(1,0)^{\prime}+E(1,0)^{\prime \prime}\right] / 2
$$

In this elementary case energy of all rays identical and in the normalized form it equal to unit. We can accept it average energy for two passes is equal to unit too. Let us write down result of our simple calculations for the first shell and energy of $\boldsymbol{E}(\mathbf{1}, \mathbf{0})$ in the form of the table in Fig.4:

$$
n=1 \stackrel{l=0}{\longrightarrow}
$$

Fig4. Results of calculations relative energy $\boldsymbol{E}(\boldsymbol{n}, \boldsymbol{l})$ extending along the links represented in Fig.3a.

Results of our calculations for the first shell of energy $\boldsymbol{E}(\mathbf{1}, \mathbf{0})$ it is possible to present in the form of histogram in Fig.5:

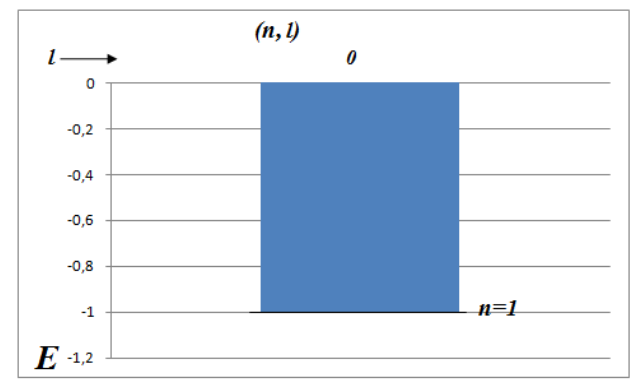

Fig5. Energy of $\boldsymbol{E}(\boldsymbol{n}, \boldsymbol{l})$, extending along the links represented in Fig.3a.

\subsubsection{Second $L$ shell of atom}

Relative value of energy extending along rays of our system in the second $L$ shell of atom (Fig.3b) at pass of $n$ is $\boldsymbol{E}(\boldsymbol{n}, \boldsymbol{l})^{\prime}$, at pass of $\mathrm{n}+1$ is $\boldsymbol{E}(\boldsymbol{n}, \boldsymbol{l})^{\prime \prime}$; and also average energy for two (n and $\mathrm{n}+1$ ) passes is $\boldsymbol{E}(\boldsymbol{n}, \boldsymbol{l})$ is calculated by means of the expressions presented in the form of tables in Fig.6:

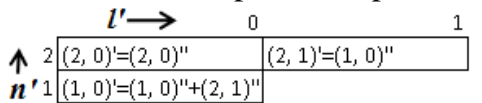

(a)

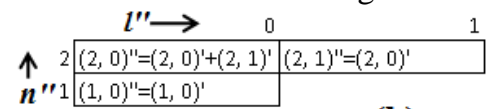

(b)

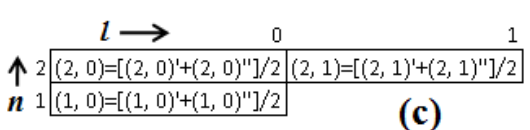

Fig.6. Formulas for calculations of relative energy of $E(n, l)^{\prime}(a), E(n, l)^{\prime \prime}(b)$, and $E(n, l)(c)$ extending along the links represented in Fig.3b.

Where for pass of $\mathrm{n}$ is $\boldsymbol{n}^{\prime}=\mathbf{1}, \mathbf{2}$ and $\boldsymbol{l}^{\prime}=\mathbf{0 , 1}$, for pass of $\mathrm{n}+1: \boldsymbol{n}^{\prime \prime}=\mathbf{1}, \mathbf{2}$ and $\boldsymbol{l}^{\prime \prime}=\mathbf{0}, \mathbf{1}$ and average energy for both passes correspond values: $\boldsymbol{n}=\mathbf{1}, \mathbf{2}$ and $\boldsymbol{l}=\mathbf{0 , 1}$.

After a large number (>100) of passes (iterations) in our system we have stationary distribution of energy in system. Let us write down result of our calculations for the second shell of energy $\boldsymbol{E}(\boldsymbol{n}, \boldsymbol{l})^{\prime}, \boldsymbol{E}(\boldsymbol{n}, \boldsymbol{l})^{\prime \prime}$ and $\boldsymbol{E}(\boldsymbol{n}, \boldsymbol{l})$, in the form of tables in Fig.7:
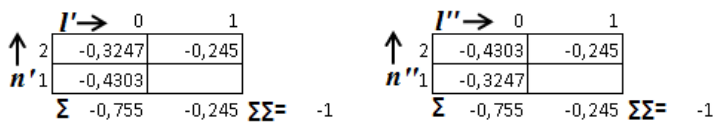

(a)

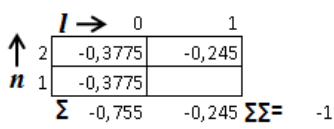

(b)

(c) 
Fig7. Results of calculations of relative energy of $\left.\boldsymbol{E}(\boldsymbol{n}, \boldsymbol{l}))^{\prime}(\boldsymbol{a}), \boldsymbol{E}(\boldsymbol{n}, \boldsymbol{l})\right)^{\prime \prime}(\boldsymbol{b})$, and $\boldsymbol{E}(\boldsymbol{n}, \boldsymbol{l})(\boldsymbol{c})$ extending along the links represented in Fig.3b after a large number of passes (iterations). In the lower part of tables the sums $(\Sigma)$ of the numbers which are written down in columns and total amount $(\Sigma \Sigma)$ are presented in tables.

In this case average energy of rays for two passes can be displayed in the form of the following schemes (histograms) of energy levels in Fig.8:

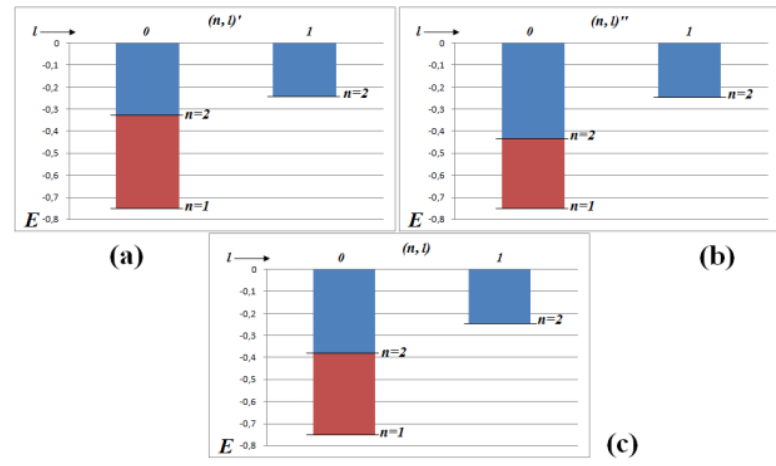

Fig8. Distribution of relative energy of $\boldsymbol{E}(\boldsymbol{n}, \boldsymbol{l})^{\prime}(\boldsymbol{a}), \boldsymbol{E}(\boldsymbol{n}, \boldsymbol{l})^{\prime \prime}(\boldsymbol{b})$, and $\boldsymbol{E}(\boldsymbol{n}, \boldsymbol{l})(\boldsymbol{c})$ extending along the links represented in Fig. $3 b$.

\subsubsection{Third $M$ shell of atom}

Relative value of energy extending along rays of our system in the third M shell of atom (Fig.3c) at pass $\mathrm{n}$ is $\boldsymbol{E}(\boldsymbol{n}, \boldsymbol{l})^{\prime}$, at pass $\mathrm{n}+1$ is $\boldsymbol{E}(\boldsymbol{n}, \boldsymbol{l})^{\prime \prime}$, and also average energy for two (n and $\left.\mathrm{n}+1\right)$ passes is $\boldsymbol{E}(\boldsymbol{n}, \boldsymbol{l})$. It is calculated by means of the expressions presented in the form of tables in Fig.9:

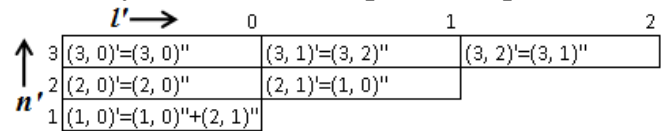

(a)

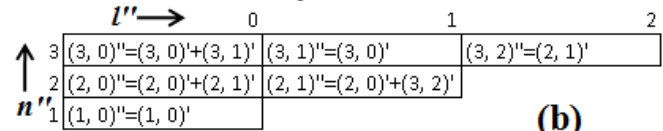

(b)

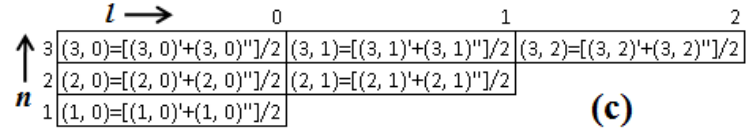

Fig9. Formulas for calculations of relative energy of $\boldsymbol{E}(\boldsymbol{n}, \boldsymbol{l})^{\prime}(\boldsymbol{a}), \boldsymbol{E}(\boldsymbol{n}, \boldsymbol{l})^{\prime \prime}(\boldsymbol{b})$, and $\boldsymbol{E}(\boldsymbol{n}, \boldsymbol{l})(\boldsymbol{c})$ extending along the links represented in Fig. $3 c$.

Where for pass of $\mathrm{n}$ we have $\boldsymbol{n}^{\prime}=\mathbf{1 , 2}, \mathbf{3}$ and $\boldsymbol{l}^{\prime}=\mathbf{0 , 1}, \mathbf{2}$; for pass of $\mathrm{n}+1$ we have $\boldsymbol{n}^{\prime \prime}=\mathbf{1 , 2 , 3}$ and $\boldsymbol{l}^{\prime \prime}=$ $\mathbf{0 , 1}, \mathbf{2}$; and for average energy for both passes we have $\boldsymbol{n}=\mathbf{1}, 2,3$ and $\boldsymbol{l}=\mathbf{0}, \mathbf{1}, 2$.

After a large number $(>100)$ of passes (iterations) in our system we have stationary distribution of energy in system. Let's write down result of our calculations for the third shell and energy of $\boldsymbol{E}(\boldsymbol{n}, \boldsymbol{l})^{\prime}(\mathrm{a}), \boldsymbol{E}(\boldsymbol{n}, \boldsymbol{l})^{\prime \prime}$ (b), and $\boldsymbol{E}(\boldsymbol{n}, \boldsymbol{l})(\mathrm{c})$, in the form of the tables in Fig.10:

\begin{tabular}{|c|c|c|c|}
\hline \multicolumn{2}{|r|}{$l^{\prime} \rightarrow 0$} & \multicolumn{2}{|c|}{$1 \quad 2$} \\
\hline & $-0,11621$ & $-0,09753$ & $-0,06318$ \\
\hline & $-0,21374$ & $-0,17939$ & \\
\hline & $-0,32995$ & & \\
\hline
\end{tabular}

(a)

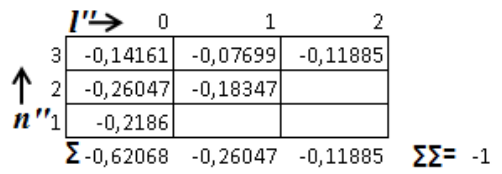

(b)

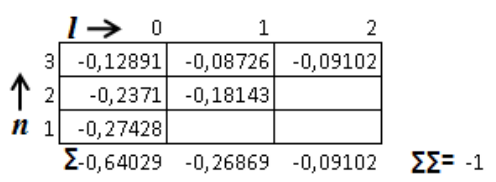

(c)

Fig10. Results of calculations of relative energy of $\boldsymbol{E}(\boldsymbol{n}, \boldsymbol{l})^{\prime}(\boldsymbol{a}), \boldsymbol{E}(\boldsymbol{n}, \boldsymbol{l}) \mathbf{~ "}^{\prime}(\boldsymbol{b})$, and $\boldsymbol{E}(\boldsymbol{n}, \boldsymbol{l})(\boldsymbol{c})$ extending along the links represented in Fig.3c after a large number of passes (iterations). In the lower part of tables the sums $(\Sigma)$ of the numbers which are written down in columns and total amount $(\Sigma \Sigma)$ are presented in tables.

In this case average energy of rays for two passes can be displayed in the form of the following schemes (histograms) of energy levels in Fig.11: 


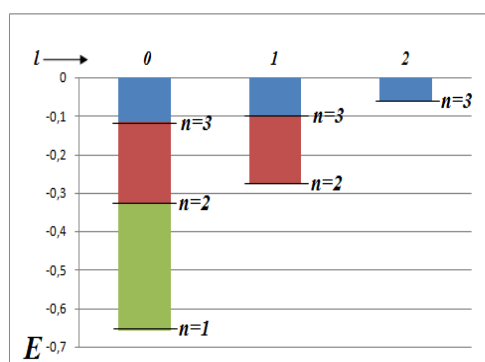

(a)

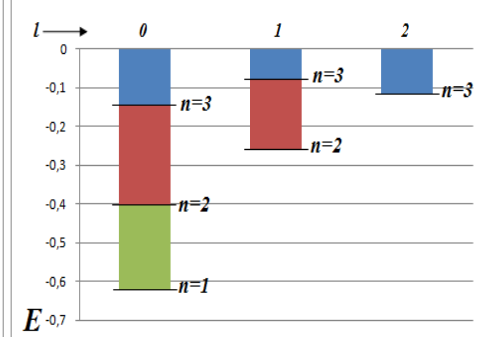

(b)

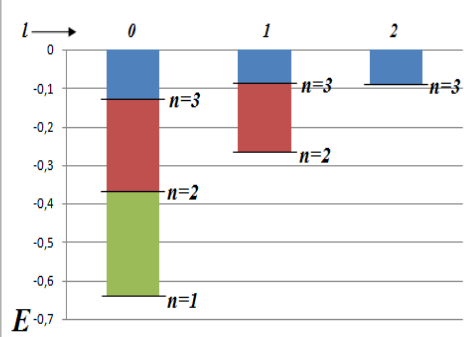

(c)

Fig11. Distribution of relative energy of $\boldsymbol{E}(\boldsymbol{n}, \boldsymbol{l})^{\prime}(\boldsymbol{a}), \boldsymbol{E}(\boldsymbol{n}, \boldsymbol{l})^{\prime \prime}(\boldsymbol{b})$, and $\boldsymbol{E}(\boldsymbol{n}, \boldsymbol{l})(\boldsymbol{c})$ extending along the links represented in Fig.3c.

\subsection{General case. Example of 15 energy levels of quantum system.}

In Fig.12 the beginning of construction (similar to Fig.1c - f, Fig.2c - f, and Fig.3a - c) of multilevel quantum system is represented.

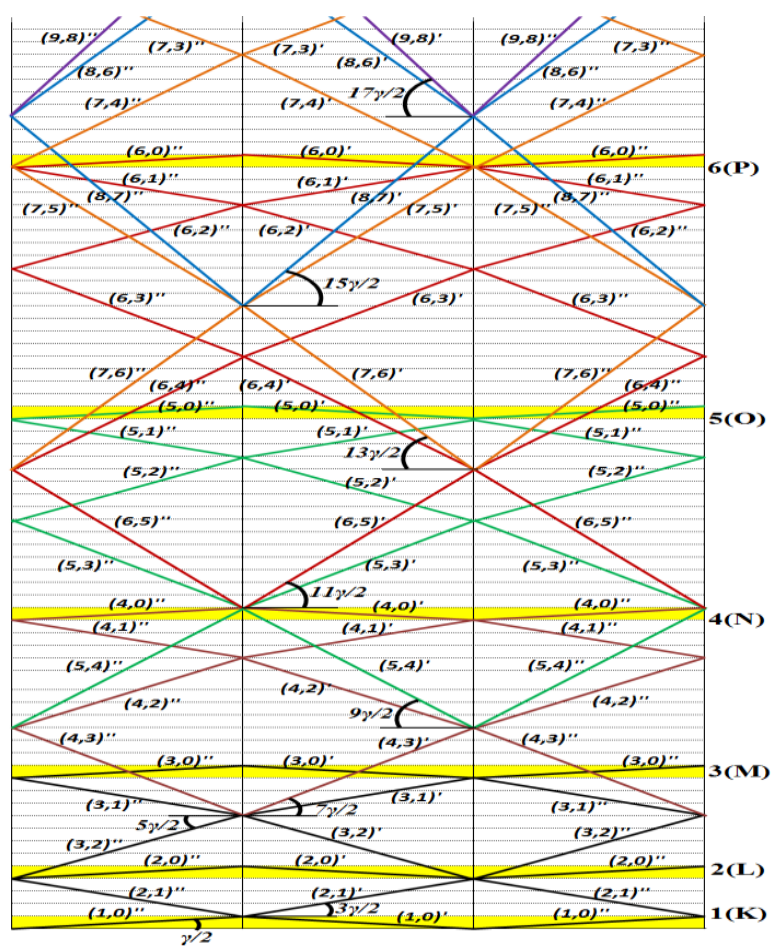

Fig12. Images of the beginning of creation of multilevel quantum system; three are shown $(n-1, n$ and $n+1)$ passes of rays in stationary system. The first $K$ shell, consists of the links inclined on angles $\gamma / 2$ to a horizontal; energy of $\boldsymbol{E}(\boldsymbol{n}, \boldsymbol{l})^{\prime}$ and $\boldsymbol{E}(\boldsymbol{n}, \boldsymbol{l})^{\prime \prime}$ (for compactness it is designated as $(\boldsymbol{n}, \boldsymbol{l})^{\prime}$ and $(\boldsymbol{n}, \boldsymbol{l})^{\prime \prime}$ ) which extends along these links are characterized by numbers $\boldsymbol{n}=\mathbf{1}$ and $\boldsymbol{l}=\mathbf{0}$. The second L shell, consists of the links inclined on angles $\gamma / 2$ and $3 \gamma / 2$ to a horizontal; energy $\boldsymbol{E}(\boldsymbol{n}, \boldsymbol{l})^{\prime}$ and $\boldsymbol{E}(\boldsymbol{n}, \boldsymbol{l})^{\prime \prime}$ which extends along these links are characterized by numbers of $\boldsymbol{n}=1,2$ and $\boldsymbol{l}=\mathbf{0}, 1$. The third $M$ shell, consists of the links inclined on angles $\gamma / 2,3 \gamma / 2$ and $5 \gamma / 2$ to a horizontal; energy of $\boldsymbol{E}(\boldsymbol{n}, \boldsymbol{l})^{\prime}$ and $\boldsymbol{E}(\boldsymbol{n}, \boldsymbol{l})^{\prime \prime}$ which extends along these links are characterized by numbers of $\boldsymbol{n}=\mathbf{1}, 2,3$ and $\boldsymbol{l}=\mathbf{0}, \mathbf{1}, \mathbf{2}$ etc. Relative value of energy extending along rays of our binomial system in multilevel quantum system (Fig.12) at pass $n$ is $\boldsymbol{E}(\boldsymbol{n}, \boldsymbol{l})^{\prime}$, at pass of $\mathrm{n}+1$ is $\boldsymbol{E}(\boldsymbol{n}, \boldsymbol{l})^{\prime \prime}$, and also average energy for two $(\mathrm{n}$ and $\mathrm{n}+1)$ passes is $\boldsymbol{E}(\boldsymbol{n}, \boldsymbol{l})$. It is calculated by means of the expressions presented in the form of tables in Fig.13: 


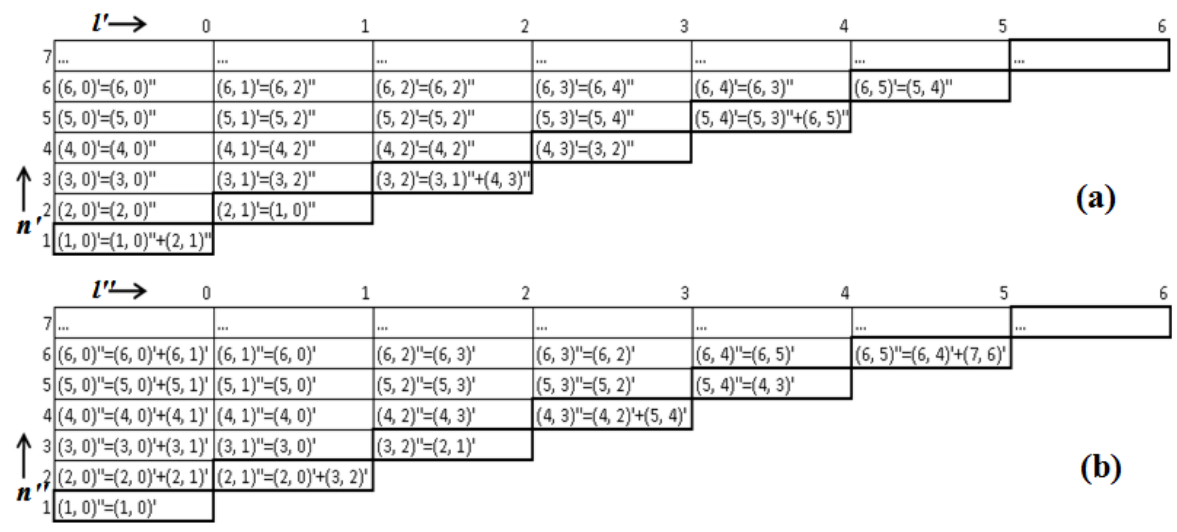

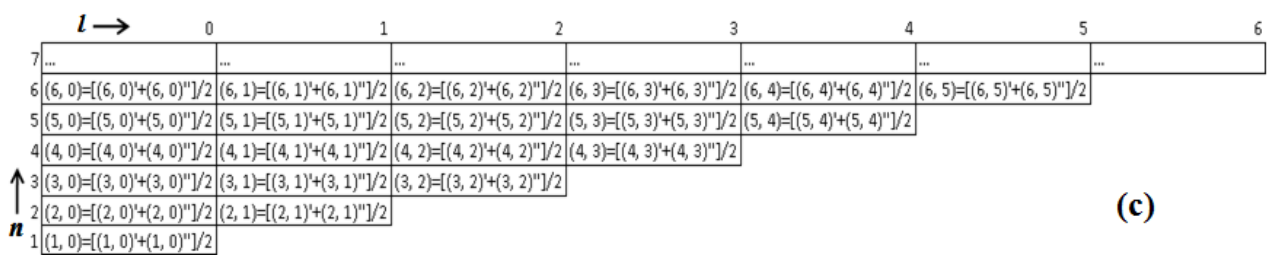

Fig13. Formulas for calculations of relative energy of $\boldsymbol{E}(\boldsymbol{n}, \boldsymbol{l})^{\prime}(\boldsymbol{a}), \boldsymbol{E}(\boldsymbol{n}, \boldsymbol{l})^{\prime \prime}(\boldsymbol{b})$, and $\boldsymbol{E}(\boldsymbol{n}, \boldsymbol{l})(\boldsymbol{c})$ extending along the links represented in Fig. 12.

Where for pass of $\mathrm{n}$ we have $\boldsymbol{n}^{\prime}=\mathbf{1}, \mathbf{2}, \mathbf{3} \ldots$, and $\boldsymbol{l}^{\prime}=\mathbf{0}, \mathbf{1}, \mathbf{2} \ldots$, for pass of $\mathrm{n}+1$ we have $\boldsymbol{n}^{\prime \prime}=\mathbf{1}, \mathbf{2}, \mathbf{3} \ldots$ and $\boldsymbol{l}^{\prime \prime}=\mathbf{0}, \mathbf{1}, \mathbf{2} \ldots$, and average energy for both passes there correspond values of $n=1,2,3 \ldots$ and $l=$ $\mathbf{0}, \mathbf{1}, \mathbf{2} \ldots$. Let us note that formulas $(13-15)$ for calculation of energy levels of the first shell of atom, also second (Fig.6) and the third (Fig.9) of shells of atom, differ from formulas for the general case a little (since the 4th shell) presented in Fig.13.

After a large number (>100) passes (iterations) of energy along links of our system we have stationary distribution of energy in the system. Let's write down result of our calculations for the quantum system consisting of 15 energy levels and energy of $\boldsymbol{E}(\boldsymbol{n}, \boldsymbol{l})^{\prime}, \boldsymbol{E}(\boldsymbol{n}, \boldsymbol{l})^{\prime \prime}$ and $\boldsymbol{E}(\boldsymbol{n}, \boldsymbol{l})$ in the form of the tables in Fig.14:

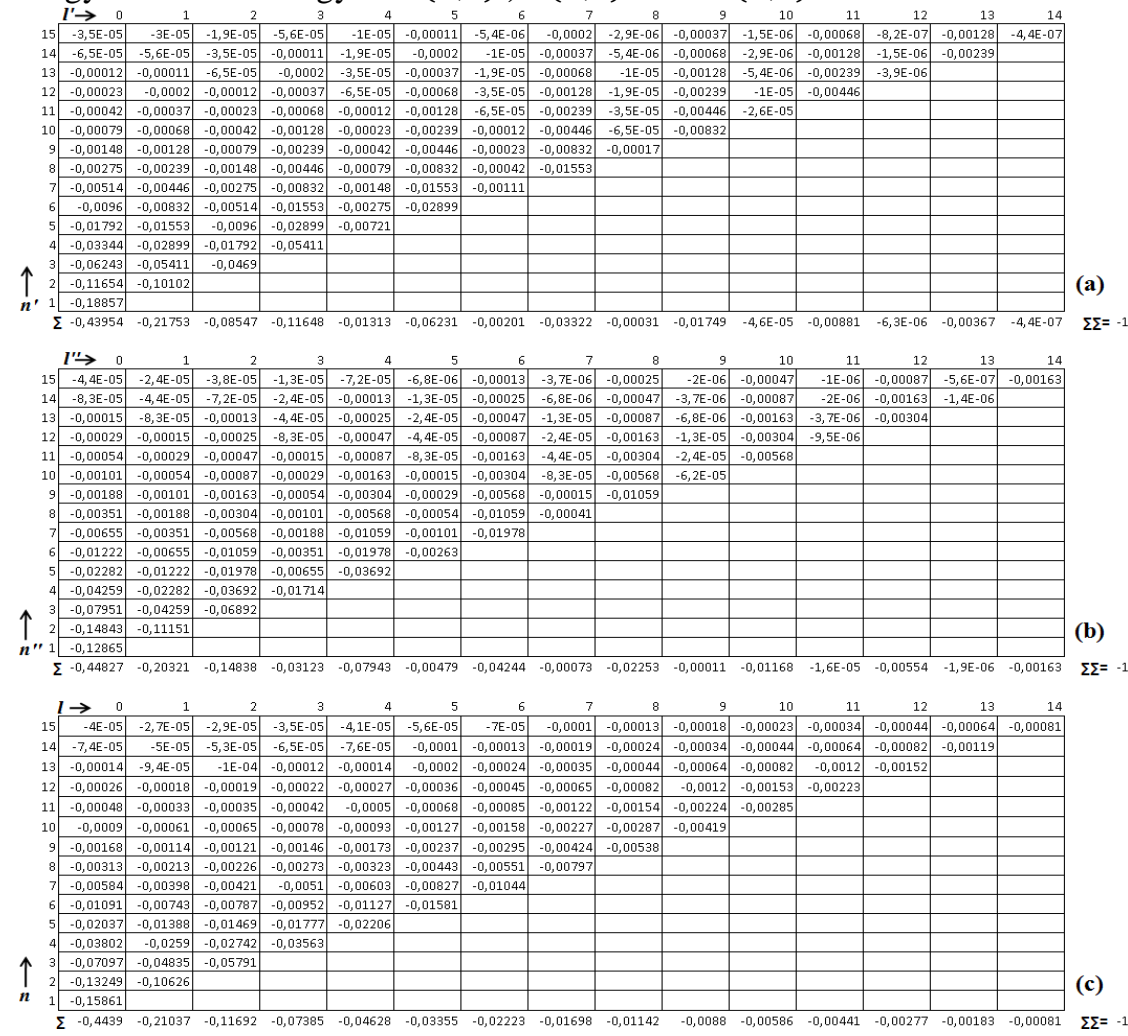


Fig14. Results of calculations of relative energy of $\boldsymbol{E}(\boldsymbol{n}, \boldsymbol{l})^{\prime}(\boldsymbol{a}), \boldsymbol{E}(\boldsymbol{n}, \boldsymbol{l})^{\prime \prime}(\boldsymbol{b})$, and $\boldsymbol{E}(\boldsymbol{n}, \boldsymbol{l})(\boldsymbol{c})$ extending along the links represented in Fig. 12 after a large number of passes (iterations). In the lower part of tables the sums $(\Sigma)$ of the numbers which are written down in columns and total amount $(\Sigma \Sigma)$ are presented in tables.

In this case average energy of rays for two passes can be displayed in the form of the following schemes (histograms) of energy levels in Fig.15:
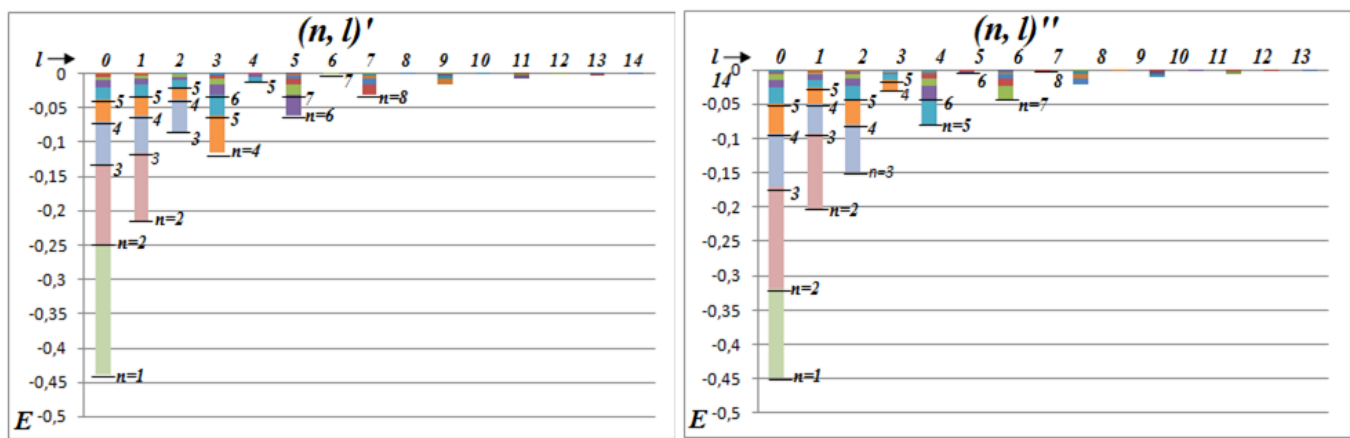

(a)

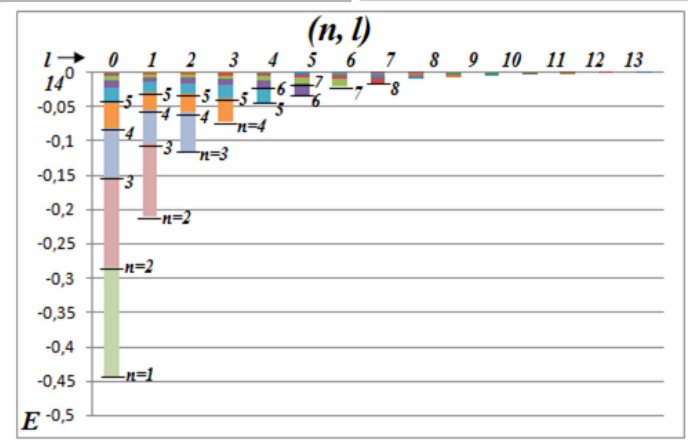

(b)

Fig15. Distribution of relative energy of $\boldsymbol{E}(\boldsymbol{n}, \boldsymbol{l})^{\prime}(\boldsymbol{a}), \boldsymbol{E}(\boldsymbol{n}, \boldsymbol{l})^{\prime \prime}(\boldsymbol{b})$, and $\boldsymbol{E}(\boldsymbol{n}, \boldsymbol{l})(\boldsymbol{c})$ extending along the links represented in Fig. 12. The similar image of spectrum is shown in Figs. 28.1, 29.1, and/or 31.1 from [3].

\subsection{Some ratios in our binomial model}

\subsubsection{Angles and quantum numbers}

In quantum mechanics [3] the condition of each electron in atom is characterized by four physical quantum numbers:

Principal $\boldsymbol{n}(n=1,2,3, \ldots)$,

Azimuthal $\boldsymbol{l}(l=0,1,2, \ldots, n-1)$,

Magnetic $\boldsymbol{m}_{\boldsymbol{l}}\left(m_{l}=-l, \ldots,-1,0,+1, \ldots,+l\right)$,

Spin $\quad \boldsymbol{m}_{\boldsymbol{s}} \quad\left(m_{s}=+\frac{1}{2},-\frac{1}{2}\right)$.

In our model of atom for the characteristic of our geometrical binomial model we used concepts of angles and distances therefore we will show compliance of the expressions (16 - 19) and our geometrical designations given earlier.

Principal $\boldsymbol{n}$ corresponds to our number $\boldsymbol{n}$ from expression (11)

Azimuthal $\boldsymbol{l}$ corresponds to our number $\boldsymbol{l}$ from expression (12)

Magnetic $\boldsymbol{m}_{\boldsymbol{l}}$ corresponds to our number $\boldsymbol{m}$ from expression (2)

Spin $\quad \boldsymbol{m}_{\boldsymbol{s}}$ corresponds to our number $\boldsymbol{s}$ from expression (4)

Let us note that expression (1) can be written also down as:

$$
p=(m+1 / 2) \gamma= \pm(l+1 / 2) \gamma \text {. }
$$

If to accept that the quantum system contains final number of electrons, then numbers in expressions $(20-22)$ can accept the minimum and maximum values $\boldsymbol{n}(\min ), \boldsymbol{n}(\max ), \boldsymbol{l}(\max )$ etc.

Values for numbers $\boldsymbol{n}, \boldsymbol{l}, \boldsymbol{m}, \boldsymbol{s}$ (expressions $20-23$ ) and also for $p$ angles (expression 24) it is possible to reduce in the separate table in Fig.16: 


\begin{tabular}{|c|c|c|c|c|}
\hline$p=s= \pm \gamma / 2$ & $p= \pm(I+1 / 2) \gamma$ & $p= \pm(2+1 / 2) \gamma$ & $\ldots$ & $p=(m+1 / 2) \gamma$ \\
\hline$l=0$ & $l=1$ & $l=2$ & $\ldots$ & $l(m a x)=|m(\max )|$ \\
\hline$n(\max )=l(\max )+1$ & $n(\max )=l(\max )+1$ & $n(\max )=l(\max )+1$ & $\ldots$ & $n(\max )=l(\max )+1$ \\
\hline$\ldots$ & $\ldots$ & $\ldots$ & $\ldots$ & \\
\hline$n=6$ & $n=6$ & $n=6$ & & \\
\hline$n=5$ & $n=5$ & $\ldots$ & & \\
\hline$\ldots$ & $\ldots$ & $n(\min )=l+l=3$ & & \\
\hline$n=2$ & $n(\min )=l+1=2$ & & & \\
\hline$n(\min )=l+1=1$ & & & & \\
\hline
\end{tabular}

Fig16. Ratios between quantum numbers from expressions (20 - 23) and angles from expression (24).

\subsubsection{Ratios between the neighbor numbers of $\boldsymbol{n}$ and $\boldsymbol{l}$}

In Fig.17 results of division of numbers of the neighbor lines (a) and neighbor columns (b), respectively, presented in Fig.14c are shown. The ratio between the neighbor values of numbers $\boldsymbol{n}$ and $\boldsymbol{l}$ accepts, the main, identical values.
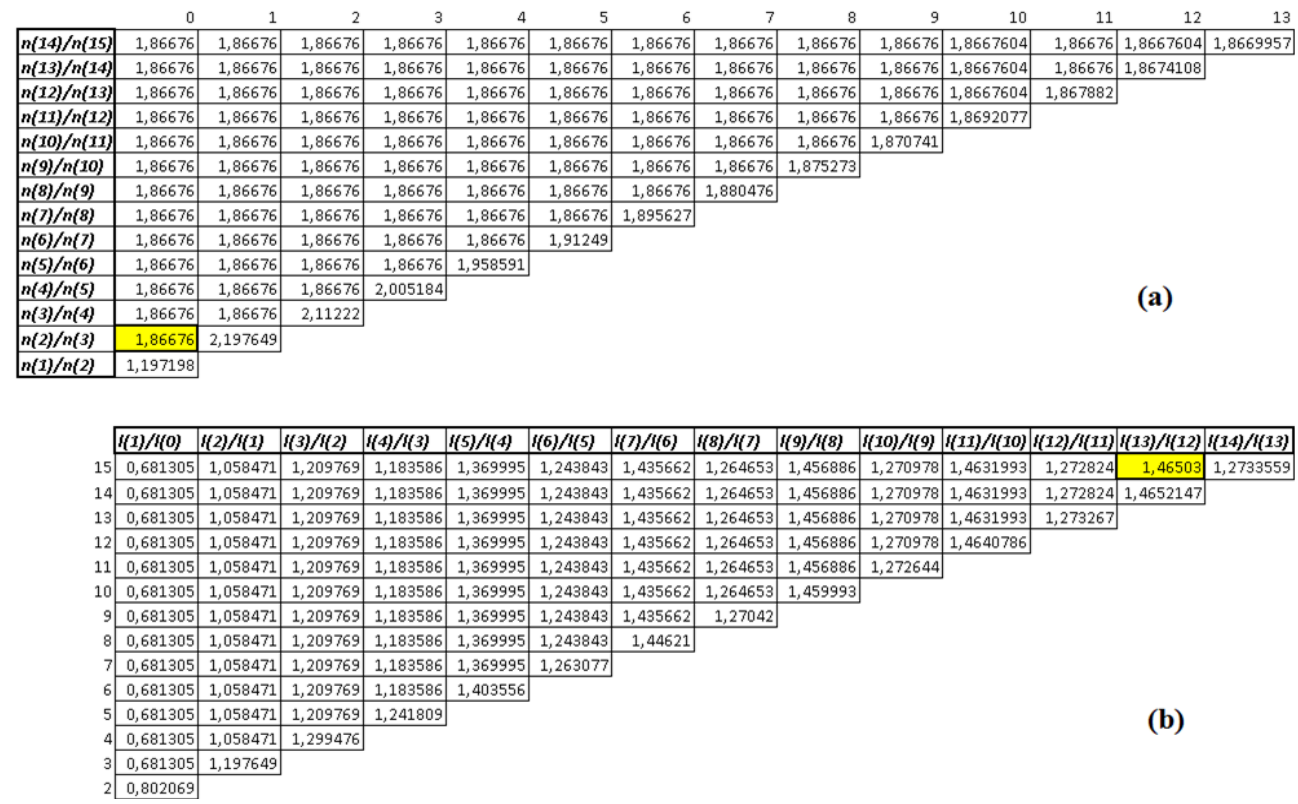

Fig17. Numerical results of division of numbers of the neighbor lines (a) and neighbor columns (b), respectively, presented in Fig.14c. So, for example, for the $l=0$ column in Fig.14c we obtain: $[\boldsymbol{n}(2) / \boldsymbol{n}(3)]=\frac{n=2}{n=3}=\frac{-0,13249}{-0,07097}=1,86676$ it is noted by a heavy yellow frame in (a). For the line $n=15$ in Fig.14c we obtain: $[\boldsymbol{l}(\mathbf{1 3}) / \boldsymbol{l}(\mathbf{1 2})]=\frac{l=13}{l=12}=\frac{-0,00064}{-0,000437}=1,46503$ it is noted by a heavy yellow frame in $(\boldsymbol{b})$, etc.

\section{ON THE GEOMETRIC INTERPRETATION OF THE SPLITTING OF SPECTRAL LINES OF} ATOM

From the experimental spectroscopic studies it is known [1 - 4] that the spectral lines of the energy levels of the atom can be single (they are called singlet) or split into several sub-levels (called multiplet). Depending on the number of sublevels of which consists multiplet we can observe doublets, triplets, quartets, quintets etc.

Most often as an example of the doublet [1 - 4] of the fine splitting of energy levels shown. The distance between the levels of the fine structure of the hydrogen atom has a value of:

or

$$
\Delta \boldsymbol{E}=\frac{\alpha^{2}}{16} \boldsymbol{E}_{\boldsymbol{i}}
$$

$$
\frac{\Delta E}{E_{i}}=\frac{\alpha^{2}}{16} \approx 3,33 * 10^{-6},
$$


Where $\boldsymbol{E}_{\boldsymbol{i}}$ is the energy of ionization of the hydrogen atom, and $\alpha$ is the dimensionless number called the fine structure constant:

$$
\alpha \approx 1 / 137 \approx 0,0073 .
$$

The appearance of fine structure is due to the presence of the electron spin. The amount of the fine splitting of light atoms does not exceed $10^{-5} \mathrm{eV}$ and strongly increases with increasing nuclear charge [4]. Various causes of atomic spectra multiplet structure described in details, for example in [1 - 4].

In this paper, we show the possibility of a geometric interpretation of the phenomenon of the multiplet splitting of the spectra within our binomial model.In the previous section we actually got an image split in two spectra of the atom in the Figs. $8(a, b) ; 11(a, b) ; 15(a, b)$ consideration of the links of the trajectories and formulas in Figs. $6(a, b) ; 9(a, b) ; 13(a, b)$.The calculation of the average value of the two parts of the doublet is shown in Figs. 8 (c); 11 (c); 15 (c) consideration of formulas in Figs. 6 (c); 9 (c); 13 (c).In the experimental spectral studies are usually first observed a single spectral line which if the resolution of the spectroscope increase it is observed as a double line (doublet) [1 -4]. In our geometric constructions on the contrary we first got two close lying energy levels (doublet) from which by computing average values we got the value of one energy level (Figs. 6(c); 9(c); 13(c)).

In the previous section we calculated the relative distribution of power when considering the stationary distribution for a pair of single ray passes: $n$ and $n+1$. However, it may likewise, we can calculate the relative distribution of energy when considering stationary distribution for the pair of double ray passes: $n-1, n$ and $n+1, n+2$. Or for a pair of triple ray passes: $n-2, n-1, n$ and $n+1, n+2, n+3$, etc.

Fig. 18 shows a second shell of the atom, and the results of calculations of energy levels:

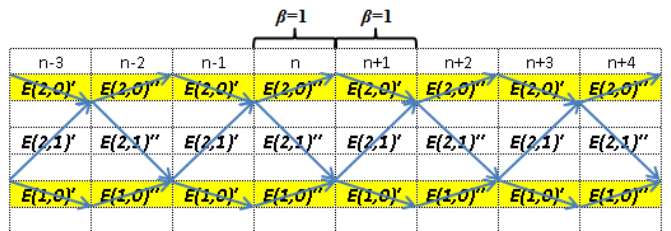

(a)

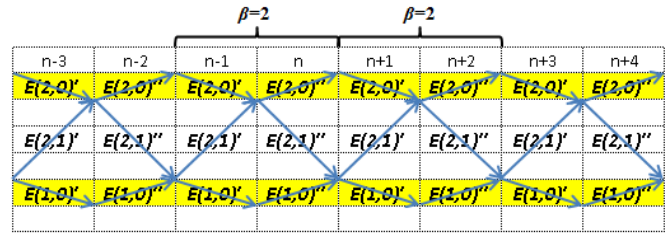

(d)

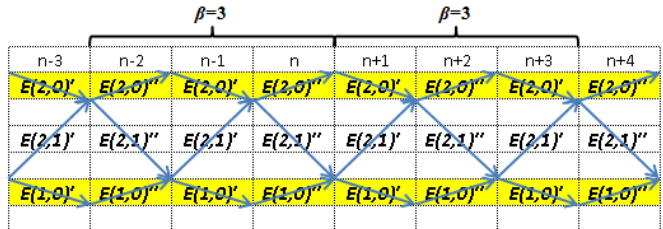

(g)

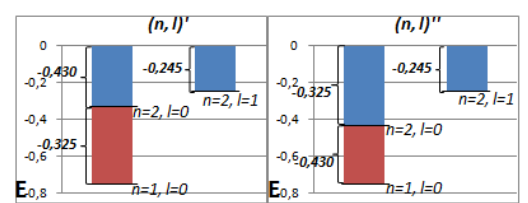

(b)

(c)

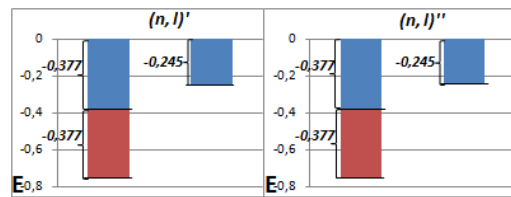

(e)

(f)

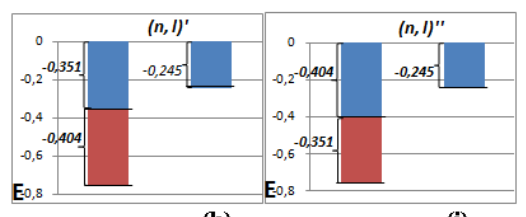

(h)

(i)

Fig18. The second shell of the atom and results of calculations of energy levels for a pair of single passes of rays $(a, b$ and $c)$, pair of double passes of rays $(d, e$ and $f)$ and pair of triple passes of the rays $(g, h$ and $i)$. (a) is similar to Fig.3(b). (b) and (c) are similar to Fig.8(a) and $(b)$ respectively. $(e)$ and $(f)$ are similar to Fig.8(c).

We consider a system of rays $\left[\boldsymbol{E}^{\prime}, \boldsymbol{E}^{\prime \prime}\right]$, (or to be specific $\left[\boldsymbol{E}^{\prime}{ }_{\mathbf{1}}, \boldsymbol{E}^{\prime \prime}{ }_{1}\right]$ ) consisting of two subsystems of rays, where $\left[\boldsymbol{E}^{\prime}\right]$ this is the first subsystem, and $\left[\boldsymbol{E}^{\prime \prime}\right]$ is the second subsystem of the system of rays.

We denote $\boldsymbol{\beta}$ as the number of consecutive passes (links) of rays, each of the subsystems used for the calculations of energy levels. In Fig. 18a $\boldsymbol{\beta}=1$, in Fig. 18b $\boldsymbol{\beta}=2$, in Fig. $18 \mathrm{c} \boldsymbol{\beta}=3$.

In the particular case the number $\boldsymbol{\beta}$ for each of the subsystems is the same.

We denote

an even number of passes (links) and

$$
\boldsymbol{\beta}=2 k
$$

$$
\boldsymbol{\beta}=2 k-1
$$

an odd number of passes (links), where $k=1,2,3, \ldots$

We replace expressions $(8-10)$ by the following designations:

$$
\boldsymbol{E}(\boldsymbol{n}, \boldsymbol{l}, \boldsymbol{\beta})^{\prime}
$$

the energy propagating along the $\boldsymbol{\beta}$ passes (links) characterized by the numbers $\boldsymbol{n}, \boldsymbol{l}$ and $\boldsymbol{\beta}$ of the first subsystem of rays $\left[\boldsymbol{E}^{\prime}\right]$ part of the system of rays $\left[\boldsymbol{E}^{\prime}, \boldsymbol{E}^{\prime \prime}\right]$. 


\section{$\boldsymbol{E}(\boldsymbol{n}, \boldsymbol{l}, \boldsymbol{\beta})^{\prime \prime}$}

the energy propagating along the $\boldsymbol{\beta}$ passes (links) characterized by the numbers $\boldsymbol{n}, \boldsymbol{l}$ and $\boldsymbol{\beta}$ of the second subsystem of rays $\left[\boldsymbol{E}^{\prime \prime}\right]$ part of the system of rays $\left[\boldsymbol{E}^{\prime}, \boldsymbol{E}^{\prime \prime}\right]$.

Mean relative value of energy for both subsystems is calculated similarly to expression (10):

$$
E(n, l, \beta)=\frac{\left[E(n, l, \beta)^{\prime}+E(n, l, \beta)^{\prime \prime}\right]}{2}=E(n, l) .
$$

If $\boldsymbol{\beta}$ is even, according to (27) then:

$$
E(n, l, \beta)^{\prime}=E(n, l, \beta)^{\prime \prime}=E(n, l, \beta)=E(n, l) .
$$

If $\boldsymbol{\beta}$ is odd, according to (27) then:

$$
E(n, l, \beta)^{\prime}=\frac{a E(n, l)+E(n, l)^{\prime}}{b}
$$

and

$$
E(n, l, \beta)^{\prime \prime}=\frac{a E(n, l)+E(n, l)^{\prime \prime}}{b},
$$

where

and

$$
\boldsymbol{a}=(\boldsymbol{\beta}-1) / 2
$$

$$
\boldsymbol{b}=(\boldsymbol{\beta}+1) / 2
$$

The relative magnitude of the distance between the two split levels (if $\boldsymbol{\beta}$ is odd) will be:

$$
\Delta E=E(n, l, \beta)^{\prime}-E(n, l, \beta)^{\prime \prime}=\frac{E(n, l)^{\prime}-E(n, l)^{\prime \prime}}{b} .
$$

Thus, even when the values of $\boldsymbol{\beta}$ our model (expression (32)) do not gives the splitting of energy levels (singlet). At odd values of $\boldsymbol{\beta}$ our model (expressions (33 - 35)) gives the splitting of energy levels (doublet).

The expression for the calculated ionization energy for our model can be written as:

$$
E_{i}=\sum_{n=1}^{n(\max )} E(n, l=0)
$$

We can except the first system of rays $\left[\boldsymbol{E}^{\prime}{ }_{1}, \boldsymbol{E}^{\prime \prime}{ }_{1}\right]$ enter an additional second system of rays $\left[\boldsymbol{E}_{2}^{\prime}, \boldsymbol{E}^{\prime \prime}{ }_{\mathbf{2}}\right]$ characterized by the numbers $\boldsymbol{n}, \boldsymbol{l}$ and $\boldsymbol{\beta}_{2}\left(\boldsymbol{\beta}_{\mathbf{2}} \neq \boldsymbol{\beta}_{1}\right)$ consisting of two subsystem of rays, where $\left[\boldsymbol{E}^{\prime}{ }_{2}\right]$ is the first subsystem and $\left[\boldsymbol{E}^{\prime \prime}{ }_{2}\right]$ is the second subsystem of ray system. Next we can enter in addition third subsystem of rays $\left[\boldsymbol{E}_{3}^{\prime}, \boldsymbol{E}^{\prime \prime}{ }_{3}\right]$ etc.

Here are a few examples.

Example 1. One system of rays $\left[\boldsymbol{E}^{\prime}, \boldsymbol{E}^{\prime \prime}\right]$ the second shell of the atom, $\boldsymbol{\beta}=1$ an odd number, the doublet, Fig. 3(b), Fig. 7, Fig. 8, Fig. 18(a-c). In accordance with the expressions (31-38) we have:

$\Delta \boldsymbol{E}=\boldsymbol{E}(\mathbf{1}, \mathbf{0}, \mathbf{1})^{\prime}-\boldsymbol{E}(\mathbf{1}, \mathbf{0}, \mathbf{1})^{\prime \prime} \approx 0,4303-0,3247=0,1056$,

$\boldsymbol{E}_{\boldsymbol{i}}=\boldsymbol{E}(\mathbf{1}, \mathbf{0}, \mathbf{1})+\boldsymbol{E}(\mathbf{2}, \mathbf{0}, \mathbf{1}) \approx 0,3775+0,3775=0,755$.

For this example: $\frac{\Delta E}{E_{i}} \approx \frac{0,1056}{0,755} \approx 0,1399$.

Example 2. One system of rays $\left[\boldsymbol{E}^{\prime}, \boldsymbol{E}^{\prime \prime}\right]$, the second shell of the atom, $\boldsymbol{\beta}=2$ an even number, singlet, Fig. 18 (d-f). In accordance with the expressions $(31,32)$ we have:

$$
E(1,0,2)^{\prime}=E(1,0,2)^{\prime}=E(2,0,2)^{\prime}=E(2,0,2)^{\prime}=E(1,0,2)=E(2,0,2) \approx 0,3775
$$

Example 3. One system of rays $\left[\boldsymbol{E}^{\prime}, \boldsymbol{E}^{\prime \prime}\right]$, the second shell of the atom, $\boldsymbol{\beta}=3$, an odd number, the doublet, Fig. $18(\mathrm{~g}-\mathrm{i})$. Example is similar to Example 1 but differs in the size of $\boldsymbol{\beta}$. In accordance with the expressions (31-38) we have: $\boldsymbol{a}=1, \boldsymbol{b}=2$,

$\Delta \boldsymbol{E}=\boldsymbol{E}(\mathbf{1}, \mathbf{0}, \mathbf{3})^{\prime}-\boldsymbol{E}(\mathbf{1}, \mathbf{0}, \mathbf{3})^{\prime \prime} \approx \frac{0,4303-0,3247}{2}=\frac{0,1056}{2}=0,0528$,

$E_{i}=E(1,0,3)+E(2,0,3) \approx 0,755$.

For this example: $\frac{\Delta E}{E_{i}} \approx \frac{0,0528,}{0,755} \approx 0,0699$. The result of the division decreased to $\boldsymbol{b}=2$ times compared with Example 1.

Example 4. One system of rays $\left[\boldsymbol{E}^{\prime}, \boldsymbol{E}^{\prime \prime}\right]$, the second shell of the atom, $\boldsymbol{\beta}=10^{5}+1$, an odd number, the doublet. The example is similar to Examples 1 and 2 but differs in the size of $\boldsymbol{\beta}$. In accordance with the expressions (31-38) we have: $\boldsymbol{a}=5 * 10^{4}, \boldsymbol{b}=5 * 10^{4}+1$,

$$
\begin{aligned}
& \Delta E=E(1,0,3)^{\prime}-E(1,0,3)^{\prime \prime} \approx \frac{0,1056}{5 * 10^{4}+1}=2,11 * 10^{-6}, \\
& E_{i}=E(1,0,3)+E(2,0,3) \approx 0,755 .
\end{aligned}
$$

For this example: $\frac{\Delta E}{\boldsymbol{E}_{\boldsymbol{i}}} \approx \frac{2,11 * 10^{6}}{0,755} \approx 2,80 * 10^{-6}$. The result of the division decreased to $\boldsymbol{b}=5 * 10^{4}+1$ times compared with Example 1, and corresponds to the order of the expression (25a).

Example 5. The combination of two systems of rays. The first system of rays $\left[\boldsymbol{E}_{\mathbf{1}}^{\prime}, \boldsymbol{E}^{\prime \prime}{ }_{\mathbf{1}}\right]$ as in Example 1, the second system of rays $\left[\boldsymbol{E}_{2}^{\prime}, \boldsymbol{E}^{\prime \prime}{ }_{2}\right]$ as in Example 2. Have both the doublet and the singlet i.e. the triplet. 
Example 6. The combination of two systems of rays. The first system of rays $\left[\boldsymbol{E}^{\prime}{ }_{\mathbf{1}}, \boldsymbol{E}^{\prime \prime}{ }_{\mathbf{1}}\right]$ as in Example 1 , the second system of rays $\left[\boldsymbol{E}_{2}^{\prime}, \boldsymbol{E}^{\prime \prime}{ }_{2}\right]$ as in Example 3. At the same time we have two different doublets i.e. the quartet.

Example 7. The combination of three systems of rays. The first system of rays $\left[\boldsymbol{E}^{\prime}{ }_{1}, \boldsymbol{E}^{\prime \prime}{ }_{\mathbf{1}}\right]$ as in Example 1 , the

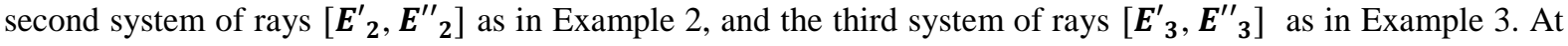
the same time we have two different doublets and singlet i.e. the quintet, etc.

Similarly, it is possible to give examples for other cases, for example, shown in Fig. $9-15$.

\section{CONCLUSIONS}

In the offered work by our geometrical and numerical researches we have shown compliance of our geometrical binomial model of atom and the energy levels of atom received experimentally from spectroscopic measurements. We have shown communication between the nuclear model of atom and wavy trajectories located along a horizontal tape. We have found the sums of power levels in the previous work [10]. In this work we have defined each of a set of power levels, and have found new regularities between them. We have characterized each of power levels by two $\boldsymbol{n}$ and $\boldsymbol{l}$ numbers which correspond to the main and azimuthal numbers of quantum system, respectively. We have shown that our new approximate model in many respects corresponds to the known models of trajectories of electrons in atom, to electronic clouds in atom and to Periodic Table. We also demonstrated calculations illustrate the splitting of the energy levels of the atom by introducing an additional parameter. Perhaps our offered model in view of works [14 - 17] can be specified further that it corresponded better with experimental spectroscopic results, by introduction additional coefficients and techniques for more exact calculation of values of energy of atom.

\section{ACKNOWLEDGMENTS}

The author expresses gratitude to S. Shnol, V. Dikusar, V. Mikhalevich, V. Smolyaninov, M. Misakyan, D. Rabonsky, J. Peters, A. Tozzi, V. Kabanov, and V. Yakimov for useful discussion and support.

\section{REFERENCES}

[1] L. D. Landau and E. M. Livshits, Theoretical physics. Vol. 3. Quantum mechanics. Nonrelativistic theory. Moscow: Nauka, 1989. (Russian)

[2] K. A. Putilov and V. A. Fabrikant, Physics course. Vol. 3. Moscow: Fizmatgiz, 1960. (Russian)

[3] I. V. Savelyev, Course of the general physics. Vol. 3. Moscow: Nauka, 1982. (Russian)

[4] D. V. Sivykhin. Atomic and Nuclear Physics. Part 1. Atomic Physics. Moscow: Nauka, 1986. (Russian)

[5] J. F. Peters, Two forms of proximal physical geometry. Axioms, sewing regions together, classes of regions, duality, and parallel fiber bundles. arXiv:1608.06208v4 [math.GN] 18 Nov 2016.

[6] Wikipedia, Alternative Periodic Tables.

[7] A. Sommerfeld, Cognition Ways in Physics. Visibility in Physics. Collection of papers, Moscow: Nauka, 1973, pp. 122,123 and others. (Russian)

[8] A. V. Yurkin, Ray trajectories and the algorithm to calculate the binomial coefficients of a new type. Proceedings of the Institute of Systems Analysis of RAS, 2009. Vol. 42, no.1, pp. 66 - 77. arXiv: 1302.4842

[9] A. V. Yurkin, Symmetric triangle of Pascal and arithmetic parallelepiped. On possibility of new evident geometrical interpretation of processes in long pipes. Lambert Academic Publishing, 2015, ISBN: 978-3-659-38411-0 (ISBN: 978-3-8443-2275-0).

[10] A. V. Yurkin, On descriptive geometrical interpretation of the principle of Pauli, elements of the table of Mendeleyev and the Newtonian laminar current of liquid. Progress in physics. Vol. 12 (2016), pp. 149-169, issue 3 (April-July).

[11] A. V. Yurkin, Silver ratios, Cardano's formula, and visual model of the second atomic shell. International Journal of Development Research. Vol. 06, Issue, 06, pp. 8077-8084, June, 2016. www.journalijdr.com

[12] A. N. Kolmogorov, I .G. Zhurbenko and A.V. Prokhorov, Introduction to the theory of probability. Moscow: Nauka, 1995. (Russian)

[13] Mathematical encyclopedic dictionary, Conformal image, Example no 1, p. 291. Moscow: Soviet Encyclopedia, 1988. (Russian)

[14] Alain Connes, Géométrie non-commutative, Université de tous les savoirs, 4, 175-190, Editions Odile Jacob, 2001.

[15] Segal, Irving (1996), Review: Noncommutative geometry, by Alain Connes, (PDF). Bull. Amer. Math. Soc. (N.S.). 33 (4): 459-465. doi: $10.1090 / \mathrm{s} 0273-0979-96-00687-8$.

[16] Penrose, Roger, The Road to Reality. Oxford University Press, 2004.

[17] Penrose, Roger, The emperor's new mind. Oxford University Press, 1989. 